\title{
The genome sequence of a soldier beetle, Cantharis rustica
}

\section{Fallén 1807 [version 1; peer review: 2 approved]}

\author{
Olga Sivell (D1), Duncan Sivell1', Natural History Museum Genome Acquisition Lab, \\ Darwin Tree of Life Barcoding collective, \\ Wellcome Sanger Institute Tree of Life programme, \\ Wellcome Sanger Institute Scientific Operations: DNA Pipelines collective, \\ Tree of Life Core Informatics collective, Darwin Tree of Life Consortium \\ ${ }^{1}$ Department of Life Sciences, Natural History Museum, London, UK
}

\begin{tabular}{l} 
V1 First published: 26 Nov 2021, 6:325 \\
https://doi.org/10.12688/wellcomeopenres.17363.1 \\
Latest published: 26 Nov 2021, 6:325 \\
https://doi.org/10.12688/wellcomeopenres.17363.1 \\
\hline
\end{tabular}

Abstract

We present a genome assembly from an individual male Cantharis rustica (a soldier beetle; Arthropoda; Insecta; Coleoptera;

Cantharidae). The genome sequence is 446 megabases in span. The majority (99.71\%) of the assembly is scaffolded into 7 chromosomal pseudomolecules, with the $X$ sex chromosome assembled.

\section{Keywords}

Cantharis rustica, sailor beetle, soldier beetle, genome sequence, chromosomal, Coleoptera

This article is included in the Tree of Life gateway.

Open Peer Review
Approval Status
Tbk, Jakarta Timur, Indonesia
University of Potsdam, Potsdam-Golm,
Germany
2. Sean Schoville iD, University of Wisconsin-
Madison, Madison, USA
Any reports and responses or comments on the
article can be found at the end of the article.


Corresponding author: Darwin Tree of Life Consortium (mark.blaxter@sanger.ac.uk)

Author roles: Sivell O: Investigation, Resources, Writing - Original Draft Preparation, Writing - Review \& Editing; Sivell D: Formal Analysis, Investigation;

Competing interests: No competing interests were disclosed.

Grant information: This work was supported by the Wellcome Trust through core funding to the Wellcome Sanger Institute (206194) and the Darwin Tree of Life Discretionary Award (218328).

The funders had no role in study design, data collection and analysis, decision to publish, or preparation of the manuscript.

Copyright: @ 2021 Sivell O et al. This is an open access article distributed under the terms of the Creative Commons Attribution License, which permits unrestricted use, distribution, and reproduction in any medium, provided the original work is properly cited.

How to cite this article: Sivell O, Sivell D, Natural History Museum Genome Acquisition Lab et al. The genome sequence of a soldier beetle, Cantharis rustica Fallén 1807 [version 1; peer review: 2 approved] Wellcome Open Research 2021, 6:325

https://doi.org/10.12688/wellcomeopenres.17363.1

First published: 26 Nov 2021, 6:325 https://doi.org/10.12688/wellcomeopenres.17363.1 


\section{Species taxonomy}

Eukaryota; Metazoa; Ecdysozoa; Arthropoda; Hexapoda; Insecta; Pterygota; Neoptera; Endopterygota; Coleoptera; Polyphaga; Elateriformia; Elateroidea; Cantharidae; Cantharinae; Cantharis; Cantharis rustica Fallén 1807 (NCBI:txid195172).

\section{Background}

Cantharis rustica (Coleoptera, Cantharidae) is a soldier beetle that can be distinguished from other British soldier beetles by its black elytra, red pronotum with a black spot, and red (or partly red) femora with the remainder of the legs black in colour (Fitton, 1973). It is common and widely distributed in southern Britain, but scarce and localised in the north (Alexander, 2003; Alexander, 2014). The species prefers lowland grassland habitats, but also occurs in woodland and other habitats with tall grass. Adults can be found on vegetation and flower heads from mid-May till the end of June (Alexander, 1991; Alexander, 2003; Fitton, 1973).

Cantharis rustica is predatory on invertebrates and has been observed to feed on a wide range of species, including Sialis lutaria (Neuroptera), Malachius bipustulatus, Adalia bipunctata, Phyllobius spp. (Coleoptera), Tenthredopsis litterata, T. nassata, Arge gracilicornis (Hymenoptera), Empis livida, Bibio marci and Scatophaga stercoraria (Diptera) (Fincher, 1951; Hobby, 1932). The adults and larvae of soldier beetles are mainly carnivorous, feeding on live and dead invertebrates, but will also feed on plant material (Alexander, 1991). The cantharid larvae have a velvety appearance and can be found in leaf litter and in the top layers of soil (Alexander, 1991; Fitton, 1973).

The karyotype of Cantharis rustica has been described and illustrated by James \& Angus (2007); males have an X0 sex chromosome system. The high-quality genome sequence described here is, to our knowledge, the first one reported for Cantharis rustica and has been generated as part of the Darwin Tree of Life project. It will aid in understanding the biology, physiology and ecology of the species.

\section{Genome sequence report}

The genome was sequenced from one male $C$. rustica collected from Wigmore Park, Luton, UK (latitude 51.88378, longitude -0.36861422). A total of 43-fold coverage in Pacific Biosciences single-molecule long reads and 48-fold coverage in 10X Genomics read clouds were generated. Primary assembly contigs were scaffolded with chromosome conformation Hi-C data. Manual assembly curation corrected 60 missing/misjoins and removed 7 haplotypic duplications, reducing the assembly length by $0.75 \%$ and the scaffold number by $72.13 \%$, and increasing the scaffold N50 by $133.18 \%$.

The final assembly has a total length of $446 \mathrm{Mb}$ in 17 sequence scaffolds with a scaffold N50 of $57.8 \mathrm{Mb}$ (Table 1). The majority, $99.71 \%$, of the assembly sequence was assigned to 7 chromosomal-level scaffolds, representing 6 autosomes (numbered by sequence length), and the $\mathrm{X}$ sex chromosome (Figure 1-Figure 4; Table 2). The assembly has a BUSCO
Table 1. Genome data for Cantharis rustica, icCanRust1.1.

\begin{tabular}{l|l|}
\hline Project accession data & \\
\hline Assembly identifier & icCanRust1.1 \\
\hline Species & Cantharis rustica \\
\hline Specimen & icCanRust1 \\
\hline NCBI taxonomy ID & NCBI:txid195172 \\
\hline BioProject & PRJEB45190 \\
\hline BioSample ID & SAMEA7524272 \\
\hline Isolate information & Male, thorax (genome \\
\hline Raw data accessions & assembly), abdomen (Hi-C) \\
\hline PacificBiosciences SEQUEL II & ERR6558187 \\
\hline 10X Genomics Illumina & ERR6054935-ERR6054938 \\
\hline Hi-C Illumina & ERR6054939 \\
\hline Genome assembly & \\
\hline Assembly accession & GCA_911387805.1 \\
\hline Accession of alternate haplotype & GCA_911387815.1 \\
\hline *B.1.2. C= complete [S= single copy, D=duplicated], F=fragmented, \\
\hline BUSCO scores is available at https://blobtoolkit.genomehubs.org/view/ \\
icCanRust1.1/dataset/CAJVQ001/busco.
\end{tabular}

v5.1.2 (Manni et al., 2021) completeness of 97.7\% (single $95.5 \%$, duplicated $2.2 \%$ ) using the endopterygota_odb10 reference set. While not fully phased, the assembly deposited is of one haplotype. Contigs corresponding to the second haplotype have also been deposited.

Chromosome 2 contains a large heterochromatic region of low confidence at approximately 20-46 Mb. This block consists of a number of scaffolds with high repeat content that can be localised to chromosome 2 but their order and orientation with respect to each other is unsure. Large islands of similar tandem repeat with high GC content are observed near both poles of Chromosome 1. Small islands of a related repeat are observed in all other chromosomes. 


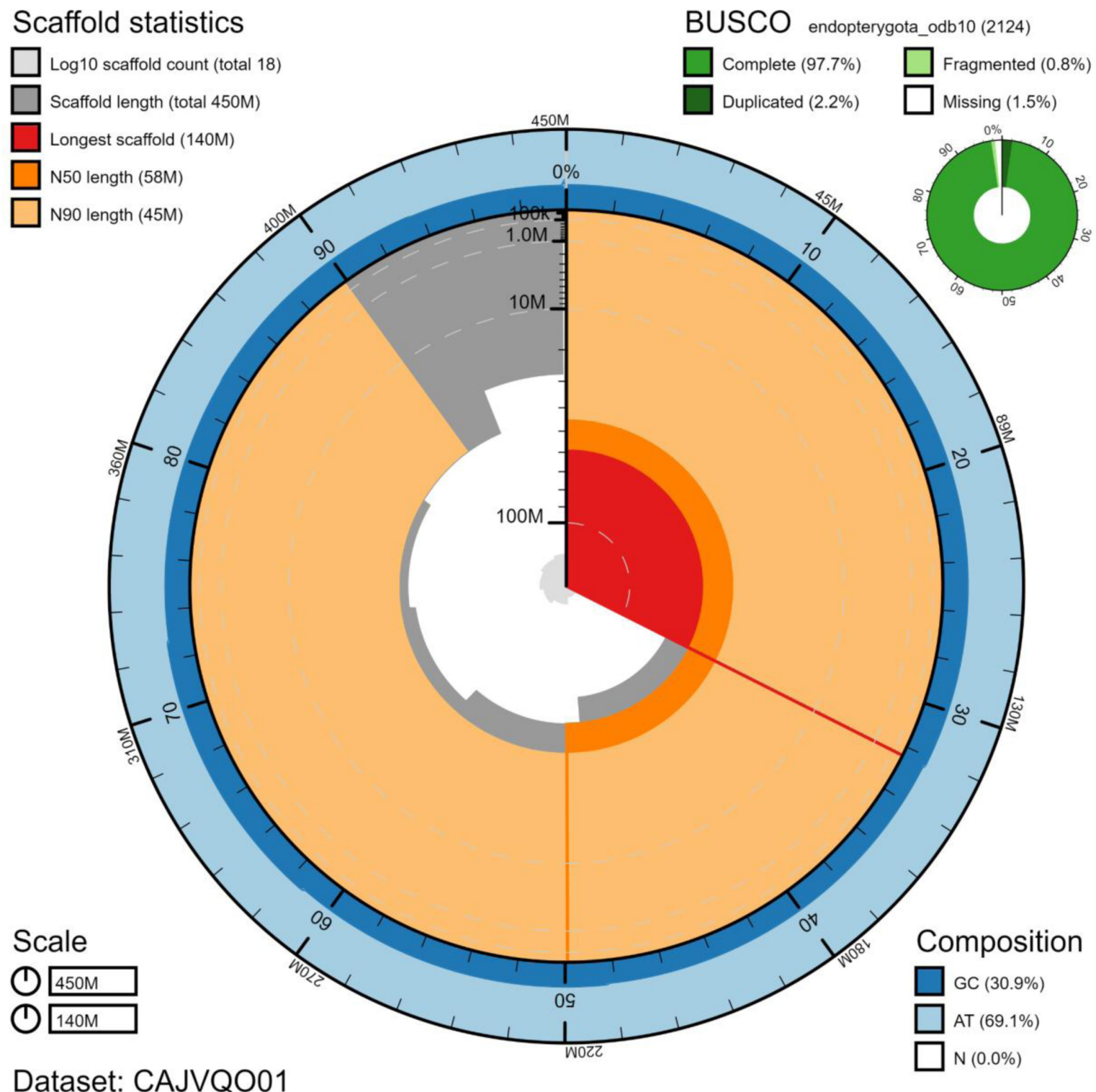

Figure 1. Genome assembly of Cantharis rustica, icCanRust1.1: metrics. The main plot is divided into 1,000 size-ordered bins around the circumference with each bin representing $0.1 \%$ of the $445,936,792$ bp assembly. The distribution of scaffold lengths is shown in dark grey with the plot radius scaled to the longest scaffold present in the assembly $(144,510,368 \mathrm{bp}$, shown in red). Orange and pale-orange arcs show the N50 and N90 scaffold lengths (57,811,309 and 44,785,338 bp), respectively. The pale grey spiral shows the cumulative scaffold count on a log scale with white scale lines showing successive orders of magnitude. The blue and pale-blue area around the outside of the plot shows the distribution of GC, AT and N percentages in the same bins as the inner plot. A summary of complete, fragmented, duplicated and missing BUSCO genes in the endopterygota_odb10 set is shown in the top right. An interactive version of this figure is available at https://blobtoolkit.genomehubs.org/view/icCanRust1.1/dataset/CAJVQ001/snail.

\section{Methods}

\section{Sample acquisition and DNA extraction}

A single female $C$. rustica (icCanRust1) was collected from Wigmore Park, Luton, UK (latitude 51.88378, longitude -0.36861422) by Olga Sivell, Natural History Museum, using a net. The sample was identified by Duncan Sivell, Natural History Museum and snap-frozen on dry ice. Unfortunately, as this specimen was collected during a COVID-19 lockdown, no image was captured prior to preservation.

DNA was extracted at the Tree of Life laboratory, Wellcome Sanger Institute. The icCanRust1 sample was weighed and dissected on dry ice with tissue set aside for Hi-C sequencing. Thorax tissue was disrupted using a Nippi Powermasher fitted with 


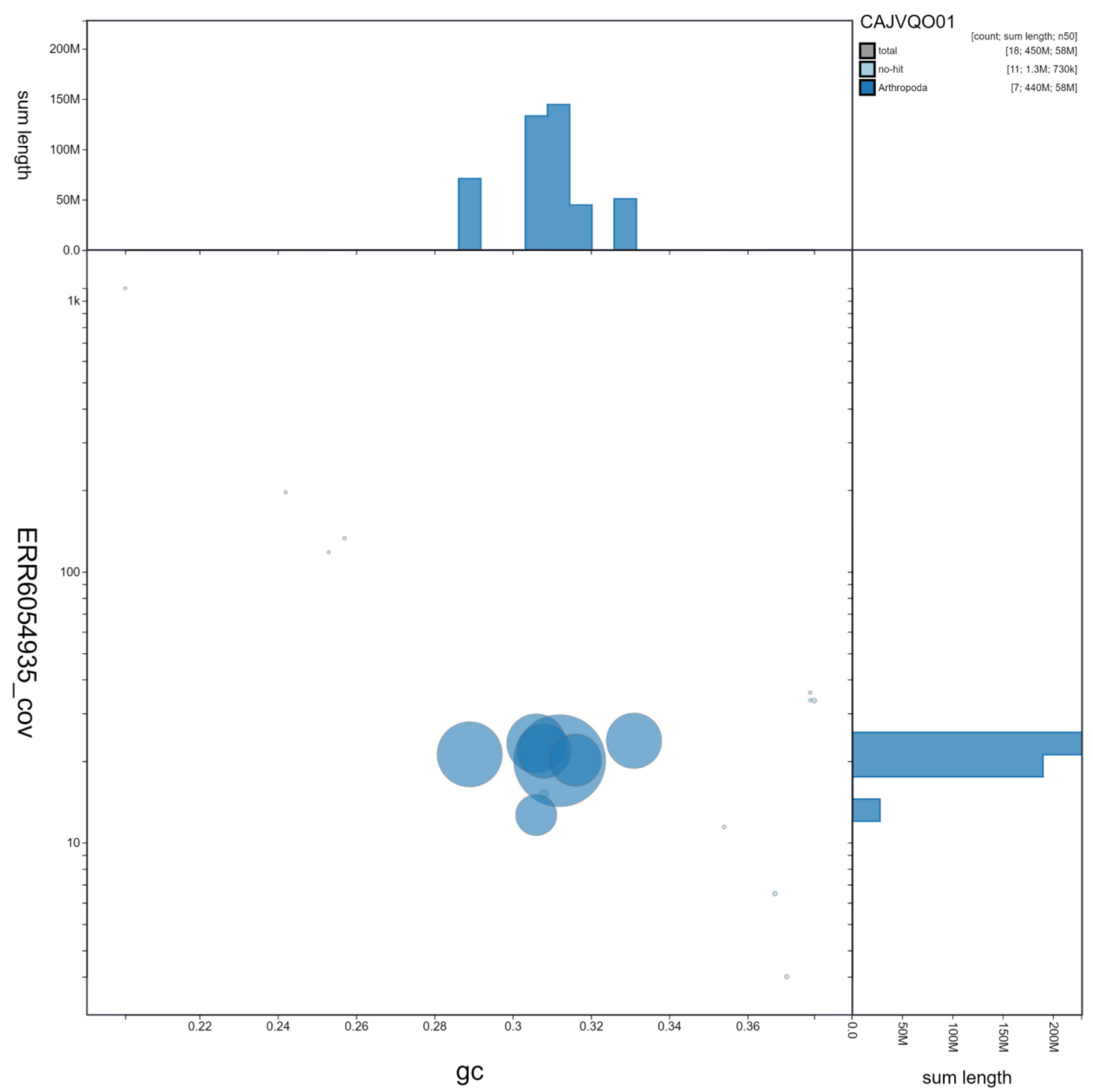

Figure 2. Genome assembly of Cantharis rustica, icCanRust1.1: GC coverage. BlobToolKit GC-coverage plot. Scaffolds are coloured by phylum. Circles are sized in proportion to scaffold length Histograms show the distribution of scaffold length sum along each axis. An interactive version of this figure is available at https://blobtoolkit.genomehubs.org/view/icCanRust1.1/dataset/CAJVQO01/blob.

a BioMasher pestle. Fragment size analysis of $0.01-0.5 \mathrm{ng}$ of DNA was then performed using an Agilent FemtoPulse. High molecular weight (HMW) DNA was extracted using the Qiagen MagAttract HMW DNA extraction kit. Low molecular weight DNA was removed from a 200-ng aliquot of extracted DNA using $0.8 \mathrm{X}$ AMpure XP purification kit prior to $10 \mathrm{X}$ Chromium sequencing; a minimum of $50 \mathrm{ng}$ DNA was submitted for $10 \mathrm{X}$ sequencing. HMW DNA was sheared into an average fragment size between $12-20 \mathrm{~kb}$ in a Megaruptor 3 system with speed setting 30. Sheared DNA was purified by solidphase reversible immobilisation using AMPure PB beads with a $1.8 \mathrm{X}$ ratio of beads to sample to remove the shorter fragments and concentrate the DNA sample. The concentration of the sheared and purified DNA was assessed using a Nanodrop 


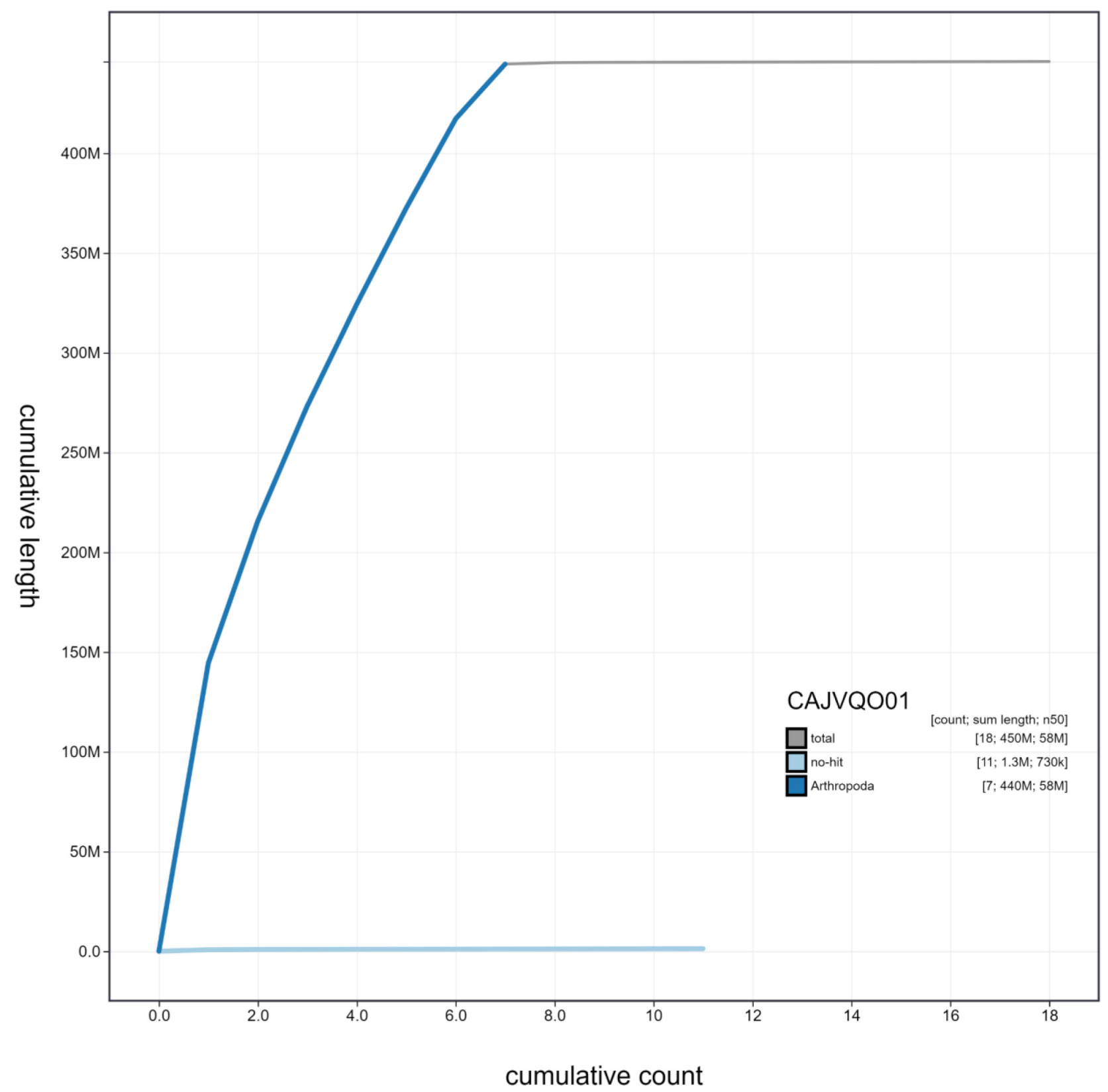

Figure 3. Genome assembly of Cantharis rustica, icCanRust1.1: cumulative sequence. BlobToolKit cumulative sequence plot. The grey line shows cumulative length for all scaffolds. Coloured lines show cumulative lengths of scaffolds assigned to each phylum using the buscogenes taxrule. An interactive version of this figure is available at https://blobtoolkit.genomehubs.org/view/icCanRust1.1/dataset/ CAJVQ001/cumulative.

spectrophotometer and Qubit Fluorometer and Qubit dsDNA High Sensitivity Assay kit. Fragment size distribution was evaluated by running the sample on the FemtoPulse system.

\section{Sequencing}

Pacific Biosciences HiFi circular consensus and 10X Genomics read cloud DNA sequencing libraries were constructed according to the manufacturers' instructions. Sequencing was performed by the Scientific Operations core at the Wellcome Sanger Instituteon Pacific Biosciences SEQUEL II and Illumina HiSeq $\mathrm{X}$ instruments. Hi-C data were generated from abdomen tissue using the Arima $\mathrm{Hi}-\mathrm{C}+$ kit and sequenced on an Illumina NovaSeq 6000 instrument.

Genome assembly

Assembly was carried out with Hifiasm (Cheng et al., 2021); haplotypic duplication was identified and removed with 


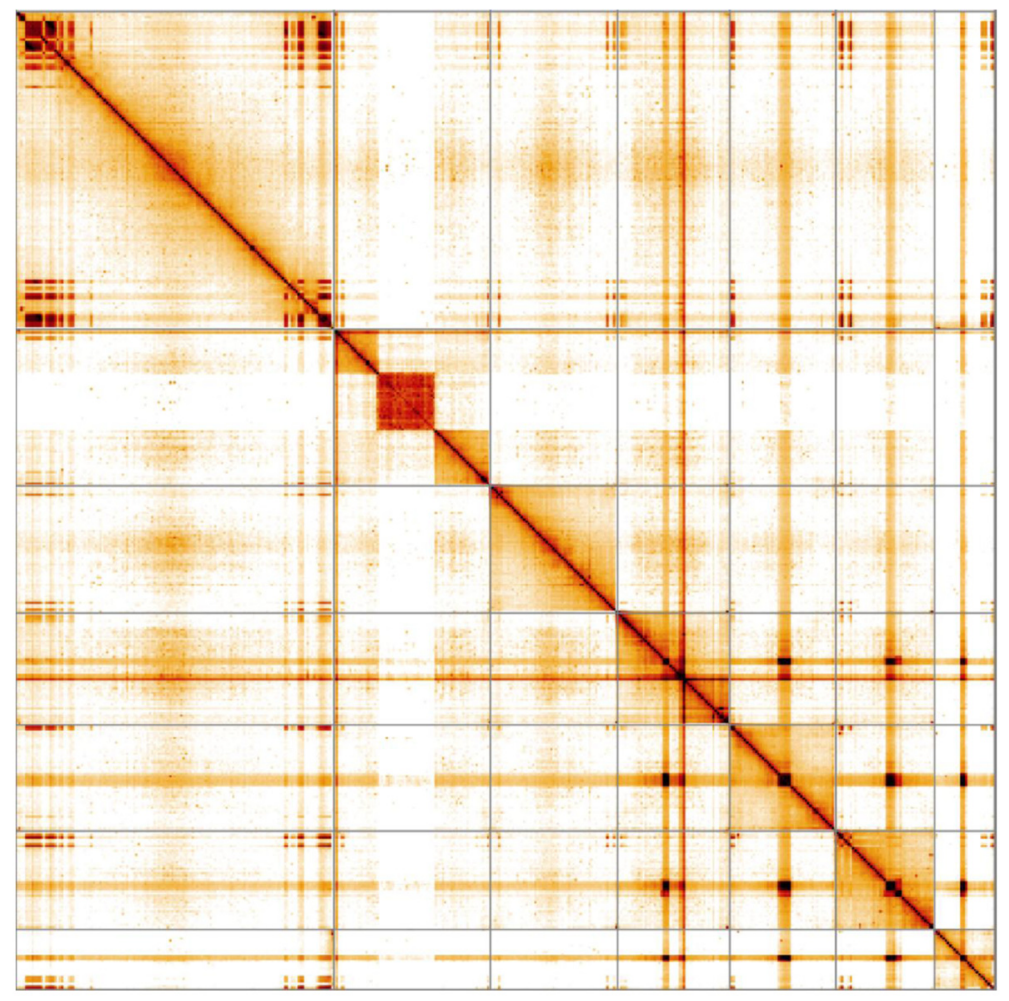

Figure 4. Genome assembly of Cantharis rustica, icCanRust1.1: Hi-C contact map. Hi-C contact map of the icCanRust1.1 assembly, visualised in HiGlass.

\section{Table 2. Chromosomal pseudomolecules in the genome assembly of Rhagonycha fulva, icRhaFulv1.1.}

\begin{tabular}{|c|c|c|c|}
\hline $\begin{array}{c}\text { INSDC } \\
\text { accession }\end{array}$ & Chromosome & Size (Mb) & GC\% \\
\hline OU426877.1 & 1 & 144.51 & 31.2 \\
\hline OU426878.1 & 2 & 71.07 & 28.9 \\
\hline OU426879.1 & 3 & 57.81 & 30.6 \\
\hline OU426880.1 & 4 & 50.92 & 33.1 \\
\hline OU426881.1 & 5 & 48.21 & 30.8 \\
\hline OU426882.1 & 6 & 44.79 & 31.6 \\
\hline OU426883.1 & $X$ & 27.33 & 30.6 \\
\hline OU426884.1 & MT & 0.02 & 20.3 \\
\hline- & Unplaced & 1.29 & 32.7 \\
\hline
\end{tabular}

purge_dups (Guan et al., 2020). One round of polishing was performed by aligning $10 \mathrm{X}$ Genomics read data to the assembly with longranger align, calling variants with freebayes (Garrison \& Marth, 2012). The assembly was then scaffolded with Hi-C data (Rao et al., 2014) using SALSA2 (Ghurye et al., 2019). The assembly was checked for contamination and corrected using the gEVAL system (Chow et al., 2016) as described previously (Howe et al., 2021). Manual curation was performed using gEVAL, HiGlass (Kerpedjiev et al., 2018) and Pretext. The mitochondrial genome was assembled using MitoHiFi (Uliano-Silva et al., 2021). The genome was analysed and BUSCO scores generated within the BlobToolKit environment (Challis et al., 2020). Table 3 contains a list of all software tool versions used, where appropriate.

\section{Ethics/compliance issues}

The materials that have contributed to this genome note have been supplied by a Darwin Tree of Life Partner. The submission of materials by a Darwin Tree of Life Partner is subject to the Darwin Tree of Life Project Sampling Code of Practice. By agreeing with and signing up to the Sampling Code of Practice, the Darwin Tree of Life Partner agrees they will meet the legal and ethical requirements and standards set out within this document in respect of all samples acquired for, and supplied to, the Darwin Tree of Life Project. Each transfer of samples is further undertaken according to a Research Collaboration Agreement or Material Transfer Agreement entered into by the Darwin Tree of Life Partner, Genome Research Limited (operating as the Wellcome Sanger Institute), and in some circumstances other Darwin Tree of Life collaborators. 


\section{Table 3. Software tools used.}

\begin{tabular}{|c|c|c|}
\hline Software tool & Version & Source \\
\hline Hifiasm & $0.12-r 304$ & Cheng et al., 2021 \\
\hline purge_dups & 1.2 .3 & Guan et al., 2020 \\
\hline SALSA2 & 2.2 & Ghurye et al., 2019 \\
\hline longranger align & 2.2 .2 & $\begin{array}{l}\text { https://support.10xgenomics. } \\
\text { com/genome-exome/software/ } \\
\text { pipelines/latest/advanced/other- } \\
\text { pipelines }\end{array}$ \\
\hline freebayes & $\begin{array}{l}\text { 1.3.1-17- } \\
\text { gaa2ace8 }\end{array}$ & Garrison \& Marth, 2012 \\
\hline MitoHiFi & 2.1 & Uliano-Silva et al., 2021 \\
\hline gEVAL & $\mathrm{N} / \mathrm{A}$ & Chow et al., 2016 \\
\hline HiGlass & 1.11 .6 & Kerpedjiev et al., 2018 \\
\hline PretextView & $0.2 . x$ & $\begin{array}{l}\text { https://github.com/wtsi-hpag/ } \\
\text { PretextView }\end{array}$ \\
\hline BlobToolKit & 2.6 .2 & Challis et al., 2020 \\
\hline
\end{tabular}

\section{Data availability}

European Nucleotide Archive: Cantharis rustica (sailor beetle). Accession number PRJEB45190; https://identifiers.org/ena.embl/ PRJEB45190.
The genome sequence is released openly for reuse. The C. rustica genome sequencing initiative is part of the Darwin Tree of Life (DToL) project. All raw sequence data and the assembly have been deposited in INSDC databases. The genome will be annotated using the RNA-Seq data and presented through the Ensembl pipeline at the European Bioinformatics Institute. Raw data and assembly accession identifiers are reported in Table 1.

\section{Author information}

Members of the Natural History Museum Genome Acquisition Lab are listed here: https://doi.org/10.5281/zenodo.4790043.

Members of the Darwin Tree of Life Barcoding collective are listed here: https://doi.org/10.5281/zenodo.4893704.

Members of the Wellcome Sanger Institute Tree of Life programme collective are listed here: https://doi.org/10.5281/ zenodo. 5377053 .

Members of Wellcome Sanger Institute Scientific Operations: DNA Pipelines collective are listed here: https://doi.org/10.5281/ zenodo.4790456.

Members of the Tree of Life Core Informatics collective are listed here: https://doi.org 10.5281/zenodo.5013542.

Members of the Darwin Tree of Life Consortium are listed here: https://doi.org/10.5281/zenodo.4783559.
Alexander K: Cantharidae. In A Coleopterist's Handbook (3rd Edition). edited by J. Cooter, The Amateur Entomologists' Society, 1991; 114-16.

Alexander KNA: Provisional Atlas of the Cantharoidea and Buprestoidea (Coleoptera) of Britain and Ireland. Centre for Ecology \& Hydrology Biological Records Centre, 2003.

Reference Source

Alexander KNA: A Review of the Beetles of Great Britain: The Soldier Beetles and Their Allies. Species Status. 2014; 16.

Challis R, Richards E, Rajan J, et al.: BlobToolKit - Interactive Quality Assessment of Genome Assemblies. G3 (Bethesda). 2020; 10(4): 1361-74. PubMed Abstract | Publisher Full Text | Free Full Text

Cheng H, Concepcion GT, Feng X, et al.: Haplotype-Resolved de Novo Assembly Using Phased Assembly Graphs with Hifiasm. Nat Methods. 2021 18(2): 170-75.

PubMed Abstract | Publisher Full Text | Free Full Text

Chow W, Brugger K, Caccamo M, et al.: gEVAL - a web-based browser for evaluating genome assemblies. Bioinformatics. 2016; 32(16): 2508-10. PubMed Abstract | Publisher Full Text | Free Full Text

Fincher F: Cantharis Rustica Fallén (Col., Cantharidae) and Its Prey. Entomologist's Monthly Magazine. 1951; 87(19).

Fitton MG: Studies on the Biology and Ecology of Cantharidae (coleoptera). 1973.

Reference Source

Garrison E, Marth G: Haplotype-Based Variant Detection from Short-Read Sequencing. arXiv: 1207.3907. 2012.

Reference Source

Ghurye J, Rhie A, Walenz BP, et al.: Integrating Hi-C Links with Assembly Graphs for Chromosome-Scale Assembly. PLOS Comput Biol. 2019; 15(8): e1007273.

PubMed Abstract | Publisher Full Text | Free Full Text

Guan D, McCarthy SA, Wood J, et al.: Identifying and Removing Haplotypic Duplication in Primary Genome Assemblies. Bioinformatics. 2020; 36(9): 2896-98.

PubMed Abstract | Publisher Full Text | Free Full Text

Hobby BM: Prey of Cantharidae (Coleoptera). Journal of Entomological Society of the South of England. 1932; 1(6).

Howe K, Chow W, Collins J, et al.: Significantly Improving the Quality of Genome Assemblies through Curation. GigaScience. 2021; 10(1): giaa153. PubMed Abstract | Publisher Full Text | Free Full Text

James LV, Angus RB: A Chromosomal Investigation of Some British Cantharidae (Coleoptera). Genetica. 2007; 130(3): 293-300.

PubMed Abstract | Publisher Full Text

Kerpedjiev P, Abdennur N, Lekschas F, et al.: HiGlass: Web-Based Visual Exploration and Analysis of Genome Interaction Maps. Genome Biol. 2018; 19(1): 125.

PubMed Abstract | Publisher Full Text | Free Full Text

Manni M, Berkeley MR, Seppey M, et al:: BUSCO Update: Novel and

Streamlined Workflows along with Broader and Deeper Phylogenetic Coverage for Scoring of Eukaryotic, Prokaryotic, and Viral Genomes. Mol Biol Evol. 2021; 38(10): 4647-54.

PubMed Abstract | Publisher Full Text | Free Full Text

Rao SSP, Huntley MH, Durand NC, et al: A 3D Map of the Human Genome at Kilobase Resolution Reveals Principles of Chromatin Looping. Cell. 2014; 159(7): 1665-80.

PubMed Abstract | Publisher Full Text | Free Full Text

Uliano-Silva M, Nunes JGF, Krasheninnikova K, et al:: marcelauliano/MitoHiFi: mitohifi v2 0. 2021.

Publisher Full Text 


\section{Open Peer Review}

\section{Current Peer Review Status:}

\section{Version 1}

Reviewer Report 09 January 2023

https://doi.org/10.21956/wellcomeopenres.19198.r53801

(c) 2023 Schoville S. This is an open access peer review report distributed under the terms of the Creative Commons Attribution License, which permits unrestricted use, distribution, and reproduction in any medium, provided the original work is properly cited.

\section{Sean Schoville}

Department of Entomology, University of Wisconsin-Madison, Madison, WI, USA

The authors describe the first genome assembly of the soldier beetle Cantharis rustica. Combining $\mathrm{HiFi}$ long reads and $\mathrm{HI}-\mathrm{C}$ data from an adult male beetle, a chromosomal-level assembly of $\sim 446$ $\mathrm{Mb}$ is produced. The genome contains six autosomes and one $\mathrm{X}$ chromosome (the species is $\mathrm{XO}$ ). Several metrics suggest that genome has a high completeness and low contamination.

There is a discrepancy in the paper as to whether this represents a male or female (line 1 in Methods says female, the abstract says male). If uncertain, perhaps examining read coverage on the $\mathrm{X}$ is useful. To this point, it is not clear in the text how the $\mathrm{X}$ has been identified.

The program "purge_dups" was used on the hifiasm assembly, although hifiasm has a similar function embedded. It may be worth reporting the change in size or other metrics associated with the additional purge, as you may be removing real genome duplication.

Could you report on heterozygosity of the genome? It is unclear what information Freebayes has added to your assembly otherwise.

A short note on the mitochondrial genome (is it complete, same gene order as canonical Coleoptera) would be helpful.

Minor revisions:

Abstract: The last sentence makes it unclear whether the $X$ chromosome is one of the seven pseudomolecules or not. I would rephrase.

On page 3- I think you don't need the qualifier "to our knowledge" as it is undoubtedly the first given a scan of the literature.

On Page 3, reference to "the $X$ sex chromsome" could be more usefully written as "the single sex chromosome (XO)". 
One Page 3, this sentence is vague "Small islands of a related repeat are observed in all other chromosomes." Is the repeat a conserved motif found repeatedly, and is it the same as the tandem repeat referred to in the preceding sentence?

Page 7, reference to "Manual curation" should be clarified as curation of the assembly, not of a gene set.

Page 8, remove "the" before RNA-seq data

Is the rationale for creating the dataset(s) clearly described?

Yes

Are the protocols appropriate and is the work technically sound?

Yes

Are sufficient details of methods and materials provided to allow replication by others? Yes

Are the datasets clearly presented in a useable and accessible format?

Yes

Competing Interests: No competing interests were disclosed.

Reviewer Expertise: Evolutionary genomics of insects.

I confirm that I have read this submission and believe that I have an appropriate level of expertise to confirm that it is of an acceptable scientific standard.

Reviewer Report 11 April 2022

https://doi.org/10.21956/wellcomeopenres.19198.r49746

(C) 2022 Apriyanto A. This is an open access peer review report distributed under the terms of the Creative Commons Attribution License, which permits unrestricted use, distribution, and reproduction in any medium, provided the original work is properly cited.

\section{Ardha Apriyanto}

${ }^{1}$ Research and Development, PT. Astra Agro Lestari Tbk, Jakarta Timur, Indonesia

2 Biopolymer Analytics, University of Potsdam, Potsdam-Golm, Germany

In this report, the authors present a genome sequence of Cantharis rustica

(Coleoptera:Cantharidae), a soldier beetle. The sequence reported is novel and contains exciting information. Also, the dataset will provide a valuable resource for the scientific community. The genome reported in this study utilized a hybrid assembly using short and long reads. As a result, it produces a very high-quality genome assembly. However, this report does not cover the annotation of the genome sequence aided by RNAseq. 
Regarding the manuscript, I found some minor mistakes that need revision:

The species' name in table 2 should be Cantharis rustica (not Rhagonycha fulva).

Also, the dataset name should be icCanRust1.1 (not icRhaFulv1.1).

All species names should be italic, for example, Cantharis rustica, in the data availability section.

In summary, the way of research reporting is very similar to its sister reports I found in this journal, which lacks variation. However, I understand that this report is part of a large genome project consortium that commonly uses a similar assembly pipeline and reporting style. I would like to recommend it for indexing with minor revision.

Is the rationale for creating the dataset(s) clearly described?

Yes

Are the protocols appropriate and is the work technically sound?

Yes

Are sufficient details of methods and materials provided to allow replication by others? Yes

Are the datasets clearly presented in a useable and accessible format? Yes

Competing Interests: No competing interests were disclosed.

Reviewer Expertise: Genome assembly, Genomics, Transcriptomics, Bioinformatics

I confirm that I have read this submission and believe that I have an appropriate level of expertise to confirm that it is of an acceptable scientific standard. 